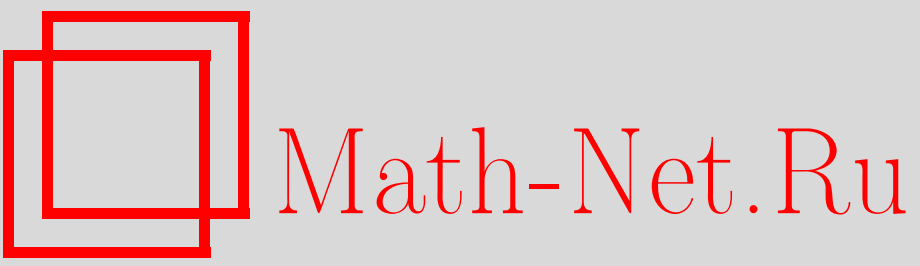

Е. Е. Скурихин, Когомологии и размерность квазиупорядоченных множеств, УМН, 2001, том 56, выпуск 1, 179-180

DOI: https://doi.org/10.4213/rm373

Использование Общероссийского математического портала Math-Net.Ru подразумевает, что вы прочитали и согласны с пользовательским соглашением

http://www . mathnet.ru/rus/agreement

Параметры загрузки:

IP : 54.198 .55 .26

26 апреля 2023 г., 13:08:40 


\title{
КОГОМОЛОГИИ И РАЗМЕРНОСТЬ КВАЗИУПОРЯДОЧЕННЫХ МНОЖЕСТВ
}

\author{
Е. Е. Скурихин
}

Целью работы является доказательство изоморфности когомологий Александрова-Чеха и Гротендика для предпучков множеств на топологизированной категории, удовлетворяющих условию типа паракомпактности. Частными случаями формулируемых результатов являются теоремы об изоморфизме обычных, а также нормальных когомологий Александрова-Чеха и Гротендика топологических пространств [1]-[3] и соответствующий результат о равномерных пространствах.

Показьвается, что ограничения на размерность $d_{\tau}$ влекут обращение в нуль когомологий Гротендика. При этом размерность $d_{\tau}$ определяется по аналогии с размерностью Лебега топологических пространств и размерностью Исбелла равномерных пространств, которые, как и нормальная размерность, являются частньми случаями $d_{\tau}$. В качестве следствий получаются резултаты об обращении в 0 пучковых когомологий на конечномерных топологических и равномерных пространствах.

Обозначения и термины содержатся в работах [4], [5]. Пусть $(K, \tau)$ - сайт, $U \in \mathrm{Ob}(\widehat{K})$. Полагаем $0=0_{U}=[\varnothing]_{\tau}^{U}$. Если не оговорено противное, то через $\lambda$ обозначается каноническая топология на $K_{U, \tau}$, так что $\alpha=\left\{U_{i} \in K_{U} \mid i \in I\right\} \in \lambda(U) \Leftrightarrow \alpha \in \tau(U)$ и все $U_{i} \tau$-замкнуты в $U$.

Будем говорить, что выполняется условие $(*)$ (соответственно $(* *))$, если $\forall \alpha=\left\{U_{i} \in K_{U, \tau}\right.$ $i \in I\} \in \tau(U) \exists \gamma^{\prime}=\left\{C_{j}^{\prime} \in\left(K_{U, \tau}\right)^{0} \mid j \in J\right\}$ - такое $\tau$-локально конечное в $U$ семейство, что $\bigvee\left\{C_{j}^{\prime} \mid j \in J\right\}=0^{\prime}$, и $\forall j \in J \exists i \in I: U_{i} \stackrel{\tau}{\supset} C_{j}^{\prime}$ в $U$ (соответственно, если вьполняется условие $(*)$ и в $\alpha$ вписывается $\tau$-локально конечное в $U \tau$-покрытие).

ОПРеДЕЛЕнИЕ 1 . Пусть $(K, \tau)$ - сайт, $U \in \mathrm{Ob}(\widehat{K}), \mathscr{B}$ - абелев предпучок на $\widehat{K}$. Напомним, что $\check{H}_{\tau}^{n}(U, \mathscr{B})=\operatorname{colim}\left\{H^{n}(\alpha, \mathscr{B}) \mid \alpha=\left\{f_{i}: D_{i} \rightarrow U \mid i \in I\right\} \in \tau(U)\right\}$, где $\left(f_{i}: D_{i} \rightarrow U\right)$ $\in \operatorname{Hom}_{\widehat{K}}\left(D_{i}, U\right), H^{n}(\alpha, \mathscr{B})$ вычисляется через коцепи Александрова-Чеха, а равенство $\alpha=$ $\left\{f_{i}: D_{i} \rightarrow U \mid i \in I\right\} \in \tau(U)$ означает, что $\bigcup\left\{f_{i}\left(D_{i}\right) \mid i \in I\right\} \quad \tau$-плотно в $U$. В частности, если $\mathscr{A} \in \mathscr{P}_{K}, \widehat{\mathscr{A}}$ - предпучок на $K_{U, \tau}$, задаваемый равенством $\widehat{\mathscr{A}}(E)=\operatorname{Hom}_{\widehat{K}}(E, \mathscr{A})$, то $\check{H}_{\lambda}^{n}(U, \widehat{\mathscr{A}})=\operatorname{colim}\left\{H^{n}(\alpha, \widehat{\mathscr{A}}) \mid \alpha \in \lambda(U)\right\}$.

Если $\mathscr{A} \in \mathscr{P}_{K}, \widehat{\mathscr{A}}$ - предпучок на $\widehat{K}$, задаваемьй равенством $\widehat{\mathscr{A}}(D)=\operatorname{Hom}_{\widehat{K}}(D, \mathscr{A})$, то в тех случаях, когда это не приводит к недоразумениям, пишем $H^{n}(\alpha, \mathscr{A})$ вместо $H^{n}(\alpha, \widehat{\mathscr{A}})$ и обозначаем $\check{H}_{\lambda}^{n}(U, \mathscr{A})=\operatorname{colim}\left\{H^{n}\left(\alpha, \mathscr{A}^{0^{\prime}}\right) \mid \alpha=\left\{U_{i} \in K_{U} \mid i \in I\right\} \in \tau(U)\right\}$.

Лемма 2. Пусть $(K, \tau)-$ сайm, $U \in \mathrm{Ob}(\widehat{K})$. Тогда

1) $\forall \mathscr{A} \in \mathscr{S}_{\tau}, \check{H}_{\lambda}^{n}(U, \mathscr{A})=\check{H}_{\lambda}^{n}(U, \widehat{\mathscr{A}})$;

2) если $K$ квазиупорядоченное множество, то

a) $\forall \mathscr{A} \in \mathscr{S}_{\tau} \quad H_{\tau}^{n}(U, \mathscr{A})=H_{\lambda}^{n}(U, \widehat{\mathscr{A}})$;

b) $\forall \mathscr{B}$ - абелева предпучка на $\widehat{K} \quad \check{H}_{\tau}^{n}(U, \mathscr{B})=\operatorname{colim}\left\{H^{n}(\alpha, \mathscr{B}) \mid \alpha=\left\{U_{i} \in K_{U} \mid\right.\right.$ $i \in I\} \in \tau(U)\}$

c) $\forall \mathscr{A} \in \mathscr{P}_{K} \quad \check{H}_{\lambda}^{n}(U, \mathscr{A})=\check{H}_{\tau}^{n}(U, \widehat{\mathscr{C}})$, где $\mathscr{C}=\mathscr{A}^{0^{\prime}}, u \forall \mathscr{A} \in \mathscr{S}_{\tau} \quad \check{H}_{\lambda}^{n}(U, \mathscr{A})=$ $\check{H}_{\lambda}^{n}(U, \widehat{\mathscr{A}})=\check{H}_{\tau}^{n}(U, \widehat{\mathscr{A}})$

Лемма 3. Пусть $(K, \tau)$ - сайт, $U \in \mathrm{Ob}(\widehat{K})$ и выполняется условие $(*)$.

1) Пусть $\mathscr{B}-U$ - $\tau$-локально нулевой предпучок на $K_{U}$, m.е. $\forall V \in K_{U}, \forall s \in \mathscr{B}(V)$, $\exists\left\{T_{i} \in K_{U} \mid i \in I\right\} \in \tau(V): s \mid T_{i}=0 \forall i \in I, \delta=\left\{W_{i} \in K_{U} \mid i \in I\right\} \in \tau(U)-\tau$-локально конечно в $U, c \in C^{n}(\delta, \mathscr{A})$, m.e. $c=\left\{c_{s} \in \mathscr{B}\left(W_{s}\right) \mid s \in I^{n+1}\right\}, W_{s}=\bigcap\left\{W_{i} \mid i \in s\right\}$, и предположим, что $\forall s \in I^{n+1}, \forall A \subset W_{s}$ из того, что $[A]_{\tau}=0_{U}$, следует, что $c_{s} \mid A=0$. Тогда $\exists \varepsilon \in \tau(U)$ и отображение $\varphi$ вписьввания $\varepsilon$ в $\delta$, такие, что $\varphi^{*} c=0$.

2) Пусть $\mathscr{B}$ - $\lambda$-нулевой предпучок на $K_{U, \tau}$, m.e. $\forall V \in K_{U, \tau}, \forall s \in \mathscr{B}(V), \exists\left\{T_{i} \in\right.$ $\left.K_{U, \tau} \mid i \in I\right\} \in \tau(V): s \mid T_{i}=0 \forall i \in I$. Пусть $\alpha=\left\{U_{i} \in K_{U, \tau} \mid i \in I\right\} \in \tau(U)-\tau$-локально 
конечно в $U, c \in C^{n}(\alpha, \mathscr{A})$, m.е. $c=\left\{c_{s} \in \mathscr{B}\left(U_{s}\right) \mid s \in I^{n+1}\right\}, U_{s}=\bigcap\left\{U_{i} \mid i \in s\right\}, u$ предположим, что $\forall s \in I^{n+1} c_{s} \mid 0_{U}=0$. Тогда $\exists \varepsilon \in \lambda(U)$ и отображение $\varphi$ вписьвания $\varepsilon$ в $\delta$, такие, чтоо $\varphi^{*} c=0$.

TEOPEма 4. Пусть $(K, \tau)-$ сайm, $U \in \mathrm{Ob}(\widehat{K})$ и выполняется условие $(* *)$. Тогда

1) $\forall \mathscr{B}-\lambda$-пучка на $K_{U, \tau}, \forall n \geqslant 0 \quad H_{\lambda}^{n}(U, \mathscr{B})=\check{H}_{\lambda}^{n}(U, \mathscr{B})$;

2) $\forall$ и: $\mathscr{A} \rightarrow \mathscr{B}$ - $\lambda$-изоморфизма предпучков на $K_{U, \tau}$ таких, что $\mathscr{A}\left(0_{U}\right)=\mathscr{B}\left(0_{U}\right)=0$, $\forall n \geqslant 0 \quad \check{H}_{\lambda}^{n}(U, \mathscr{A}) \rightarrow \check{H}_{\lambda}^{n}(U, \mathscr{B})$ является изоморфизмом;

3) если $K$ квазиупорядоченное множество, то

a) $\forall \mathscr{A} \in \mathscr{S}_{\tau}, \forall n \geqslant 0 \quad \check{H}_{\tau}^{n}(U, \widehat{\mathscr{A}})=\check{H}_{\lambda}^{n}(U, \mathscr{A})=H_{\lambda}^{n}(U, \widehat{\mathscr{A}})=H_{\tau}^{n}(U, \mathscr{A})$;

b) если $A \in K_{U} u \forall \mathscr{I} \in \mathscr{S}_{\tau}-$ ингективного, $\forall k \geqslant 1 H_{\tau}^{k}\left(U, \mathscr{I}_{A^{\prime}}\right)=0$ и $\mathscr{I}_{-}$ $U$ - $\left(0^{\prime}, A^{\prime}\right)$-мягок, то $\forall \mathscr{A} \in \mathscr{S}_{\tau} \operatorname{colim}\left\{H_{\tau}^{n}(V, \mathscr{A}) \mid V \stackrel{\tau}{\supset} A^{\prime}\right.$ в $\left.U\right\}=\check{H}_{\lambda}^{n}\left(U, \mathscr{A}_{A^{\prime}}\right)$ при $n \geqslant 0 ;$ если при этом $U \in K_{1}$, то перечисленнье условия на инвективные пучки могут быть опущень, так как они следуют из (**).

ОПРЕДЕЛЕнИЕ 5 . Пусть $(K, \tau)$ - сайт, $U \in \mathrm{Ob}(\widehat{K}), A \in \widehat{K}_{U, \tau}$. $A^{\prime}$-кратностью семейства $\alpha=\left\{U_{i} \in K_{U} \mid i \in I\right\}$ назовем наиболшшее число $m$, удовлетворяющее условию: $\forall \sigma \subset I$ из того, что мощность $\sigma$ больше $m$, следует, что $\left\{\left\{U_{i} \mid i \in \sigma\right\} \subset A\right.$. Кратностью $\alpha=\left\{U_{i} \in K_{U} \mid i \in I\right\}$ назовем $0_{U}^{\prime}$-кратность. Положим $d_{\tau} U \leqslant m \Leftrightarrow$ в любое $\tau$-покрытие $U$ можно вписать $\tau$-покрытие $U$ кратности $\leqslant m+1$, и $d_{\tau}^{U} A^{\prime} \leqslant m \Leftrightarrow$ в любое $\tau$-покрытие $U$ можно вписать $\tau$-покрытие $U A^{\prime}$-кратности $\leqslant m+1$. Очевидно, $d_{\tau}^{U} 0^{\prime}=d_{\tau} U$.

Пункты 1) и 2) нижеследующей леммы доказываются непосредственно, а 3) получается из рассуждений работы Серра [6].

Лемма 6. Пусть $(K, \tau)-$ caŭm, $U \in \mathrm{Ob}(\widehat{K}), A \in \widehat{K}_{U, \tau}$.

1) $A^{\prime}$-кратность $\alpha=\left\{U_{i} \in K_{U} \mid i \in I\right\}$ равна $A^{\prime}$-кратности $[\alpha]=\left\{\left[U_{i}\right]_{\tau}^{U} \in K_{U, \tau} \mid\right.$ $i \in I\}$;

2) $d_{\tau}^{U} A^{\prime}=d_{\lambda}^{U} A^{\prime}$, в частности, $d_{\tau} U=d_{\lambda} U$;

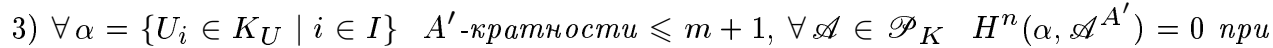
$n>m$. В частности, $\forall \alpha=\left\{U_{i} \in K_{U} \mid i \in I\right\}$ кратности $\leqslant m+1, \forall \mathscr{A} \in \mathscr{P}_{K}$ такого, что $\mathscr{A}^{0}=0, H^{n}(\alpha, \mathscr{A})=0$ npu $n>m$.

Комбинируя сфформулированные выше результаты, получаем следующую теорему.

TeOpema 7. Пусть $(K, \tau)-c a u ̈ m, U \in \mathrm{Ob}(\widehat{K}), A \in \tilde{K}_{U, \tau}$. Tогдa:

1) Ecлu d $d_{\tau}^{U} A^{\prime} \leqslant m, m o \forall \mathscr{A} \in \mathscr{P}_{K} \check{H}_{\lambda}^{n}\left(U, \mathscr{A}^{A^{\prime}}\right)=0$, a еслu $\mathscr{A}^{A}=0$, mo $\check{H}_{\lambda}^{n}(U, \widehat{\mathscr{A}})=0$ при $n>m$. В частности, $\forall \mathscr{A} \in \mathscr{S}_{\tau}, \forall n>m \quad \check{H}_{\lambda}^{n}\left(U, \mathscr{A}_{A^{\prime}}\right)=0$.

2) $E$ cлu $d_{\tau} U \leqslant m, \mathscr{A} \in \mathscr{P}_{K}$, mo $\check{H}_{\lambda}^{n}(U, \mathscr{A})=0$, a ecлu $\mathscr{A}^{0}=0$, mo $\check{H}_{\lambda}^{n}(U, \widehat{\mathscr{A}})=0$ npu $n>m$.

3) Пусть $K-$ квазиупорядоченное множество и выполняется условие (**).

a) $E c л u d_{\tau}^{U} A^{\prime} \leqslant m$, mo $\forall \mathscr{A} \in \mathscr{S}_{\tau} H_{\lambda}^{n}\left(U, \mathscr{A}_{A^{\prime}}\right)=H_{\tau}^{n}\left(U, \mathscr{A}_{A^{\prime}}\right)=0$ npu $n>m$. Ecлu при этом $U \in K_{1}$, mо $\operatorname{colim}\left\{H_{\tau}^{n}(V, \mathscr{A}) \mid V \stackrel{\tau}{\supset} A^{\prime}\right.$ в $\left.U\right\}=0$ nри $n>m$.

b) Ecлu $d_{\tau} U \leqslant m$, mo $\forall \mathscr{A} \in \mathscr{S}_{\tau} H_{\lambda}^{n}(U, \mathscr{A})=H_{\tau}^{n}(U, \mathscr{A})=0$ npu $n>m$.

\section{СПИСОК ЛИТЕРАТУРЫ}

[1] Р. Годеман. Гомологическая алгебра и теория пучков. М.: ИЛ, 1961. [2] Е. Е. Скурихин // УМН. 1980. Т. 35. № 3. С. 224-226. [3] Е. Е. Скурихин. Пучковые когомологии и полные брауэровы решетки. Владивосток: Дальнаука, 1993. [4] Е. Е. Скурихин // УМН. 1998. Т. 53. №6. С. 263-264. [5] Е. Е. Скурихин // УМН. 1999. Т. 54. № 2. С. 187-188. [6] J.-P. Serre // Ann. of Math. 1955. T. 61. C. 197-278. 